\title{
Tricuspid perivalvular leak closure through the open cells of Sapien 3 transcatheter heart valve
}

Keywords: transcatheter valve, valvular disease, perivalvular leak, central leak

\section{Imaging vignette}

\section{Background}

Recent years have seen significant developments in transcatheter valve replacement techniques. At the same time, the anatomy of the transcatheter prosthetic valve poses a new set of challenges when dealing with procedural complications, paving the way for further innovation. We present an imaging vignette that highlights some of the advances in this minimally invasive modality to treat valvular disease.

A 73-year-old female with mechanical mitral valve, tricuspid annuloplasty (32mm Edwards Physio Tricuspid incomplete ring) underwent Transcatheter Tricuspid Valve Replacement (TTVR) using off-label 29mm Edwards Sapien 3 (S3) Transcatheter Heart Valve (THV). Immediate post valve deployment, mild Perivalvular Leak (PVL) was noticed at the septal aspect (area of incomplete annuloplasty ring). Eighteen months later, patient presented with recurrent right heart failure and paracentesis due to severe tricuspid PVL (Figure 1A-C, Video 1). Percutaneous PVL closure under general anesthesia, Trans-esophageal Echocardiography (TEE) was performed. Via femoral venous access, defect was easily crossed with Agilis steerable sheath, multipurpose catheter and 0.035 " glide wire. Despite recurrent attempts, wire came through the PVL but then traversed through open cells of S3 into the right ventricle (Figure 2A), confirmed with Armada $6 \mathrm{~mm}$ balloon waist at the S3 cage (Figure 2B) (despite inflating $28 \mathrm{~mm}$ $\mathrm{Z}$ med balloon inside S3 cage, Figure $2 \mathrm{C}$ ). We decided to partially deploy the plug and assess valve function. Using 7.5F Asahi Eaucath multipurpose guide, a $12 \mathrm{~mm}$ Amplatzer ${ }^{\mathrm{TM}}$ Vascular plug II (AVP II)
Volume II Issue 3 - 202I

\author{
Muhammad Abdulbasit, ${ }^{2}$ Mark Kozak,' \\ Brandon Peterson,' Pradeep Yadav,' Tariq A \\ Ahmad' \\ 'Department of Cardiovascular Medicine, Penn State Milton \\ Hershey Medical Center, USA \\ ${ }^{2}$ Department of Nephrology, Penn State Milton Hershey Medical \\ Center, USA
}

\begin{abstract}
Correspondence: Muhammed Abdulbasit, Department of Nephrology, Penn State Milton Hershey Medical Center, Hershey, PA - USA, Tel 7I7-53I-8I56,

Email a.basit39@gmail.com
\end{abstract}

Received: May 31, 202I | Published: June 14, 202 I

was advanced through the defect. The ventricular disc was opened inside the S3 cage while body in the PVL defect (outside the S3 cage) and atrial disc on the atrial side of PVL (Figure 3A-B). With S3 function unaffected, no central leak and minimal gradient, PVL reduced to mild severity (Figure 4A-D, Video 2); the AVP II was successfully deployed (Figure 5). At 3-month follow up, there was an excellent symptomatic improvement (NYHA functional class I), with no heart failure re-hospitalization or paracentesis. Deployment of plug disc inside the valve frame is not recommended due to fear of interference with leaflet function and possible injury in long term. There was no immediate issue with the valve function in this case (due to space between the leaflet and frame of S3 and depends on size of plug used). Valve-in-Valve (S3-in-S3) would have certainly sealed all open cells treating this PVL but is more expensive option.

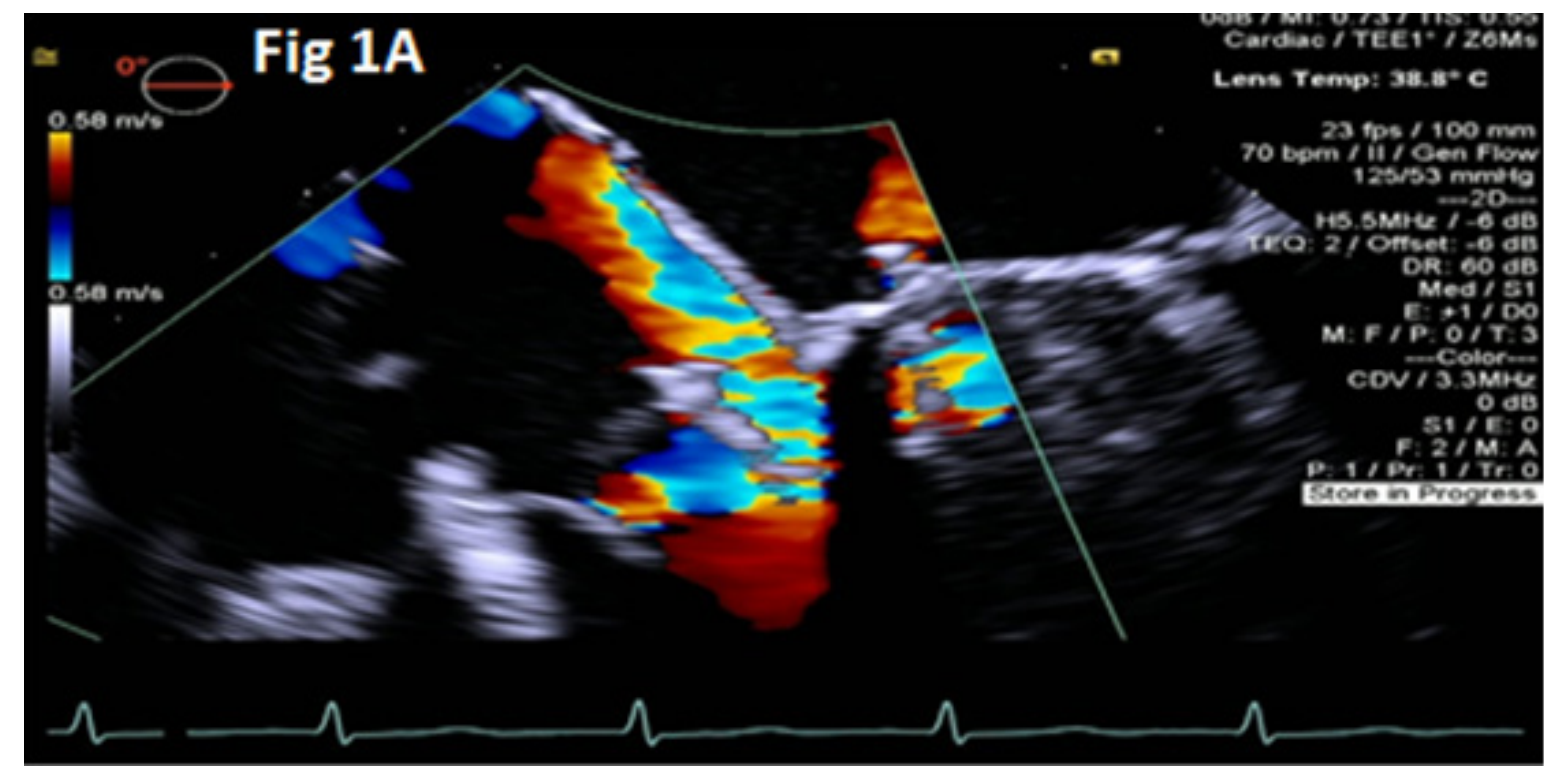



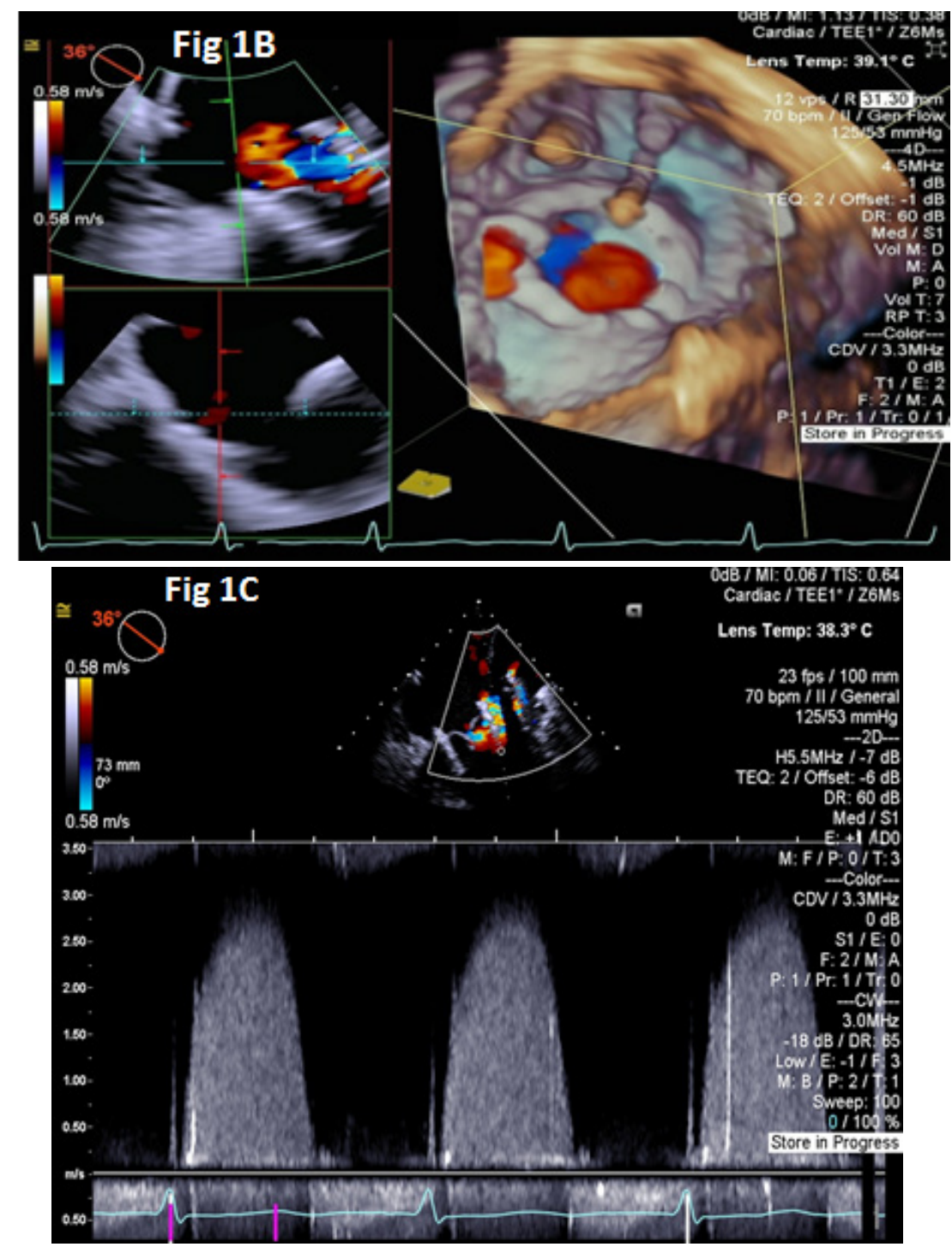

Figure I Para-valvular leak shown by color Doppler (IA) on 2D and 3D (IB) images, along with dense tricuspid spectral Doppler signal with peak velocity of $3 \mathrm{~m} / \mathrm{s}(I C)$.

Video I Mid esophageal view showing paravalvular leak on septal side of the Sapien 3 valve.

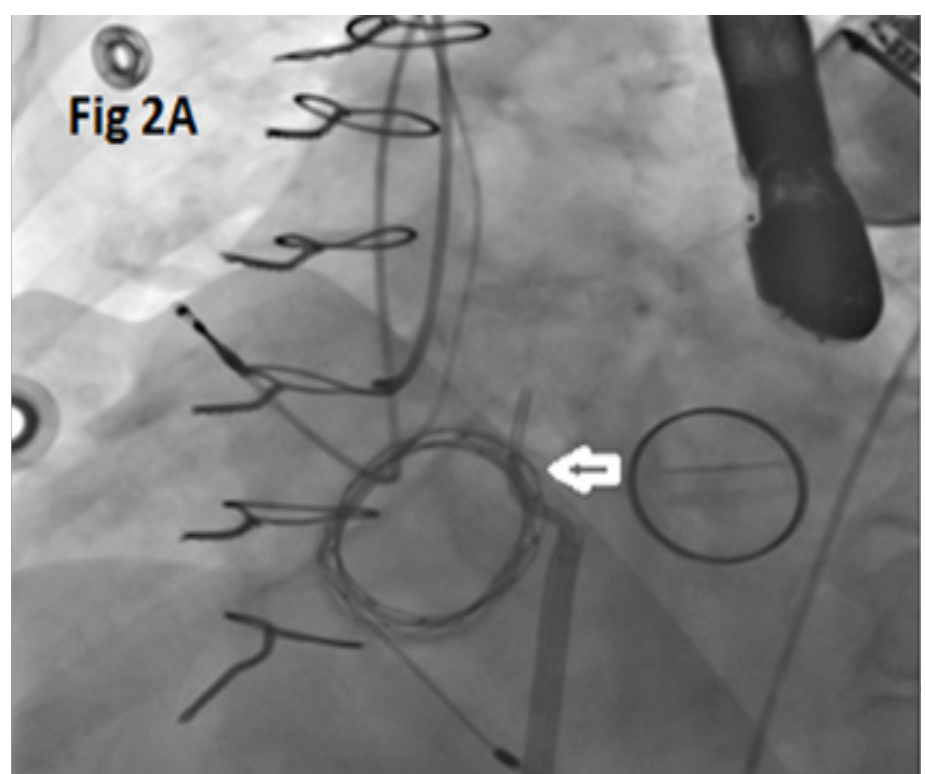

Citation: Abdulbasit M, Kozak M, Peterson B, et al. Tricuspid perivalvular leak closure through the open cells of Sapien 3 transcatheter heart valve. MOJ Clin Med Case Rep. 202I;I I(3):79-83. DOI: 10.15406/mojcr.202I.II.00387 

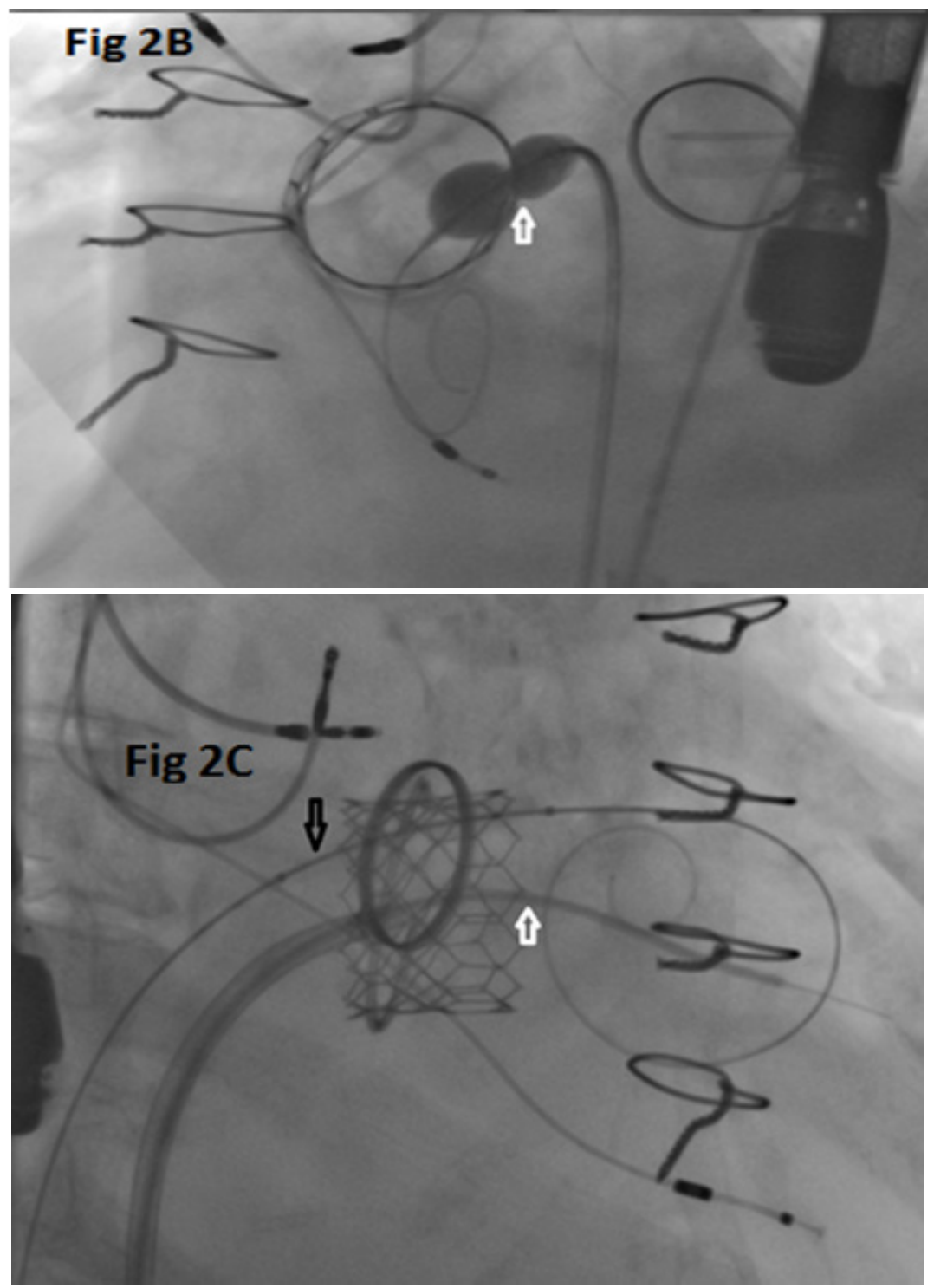

Figure 2 Multi-purpose diagnostic catheter (MPA) crossing through the PVL proximally and through the S3 cells distally (A), balloon inflation over a wire passed through the PVL channel confirms the position of hour-glass shaped inflated balloon with proximal half outside \& distal half inside the S3 cage and waist in the middle (B), along with failed attempt to cross the MPA (white arrow in C) with Safari wire along with Zmed balloon inside the S3 (black arrow).

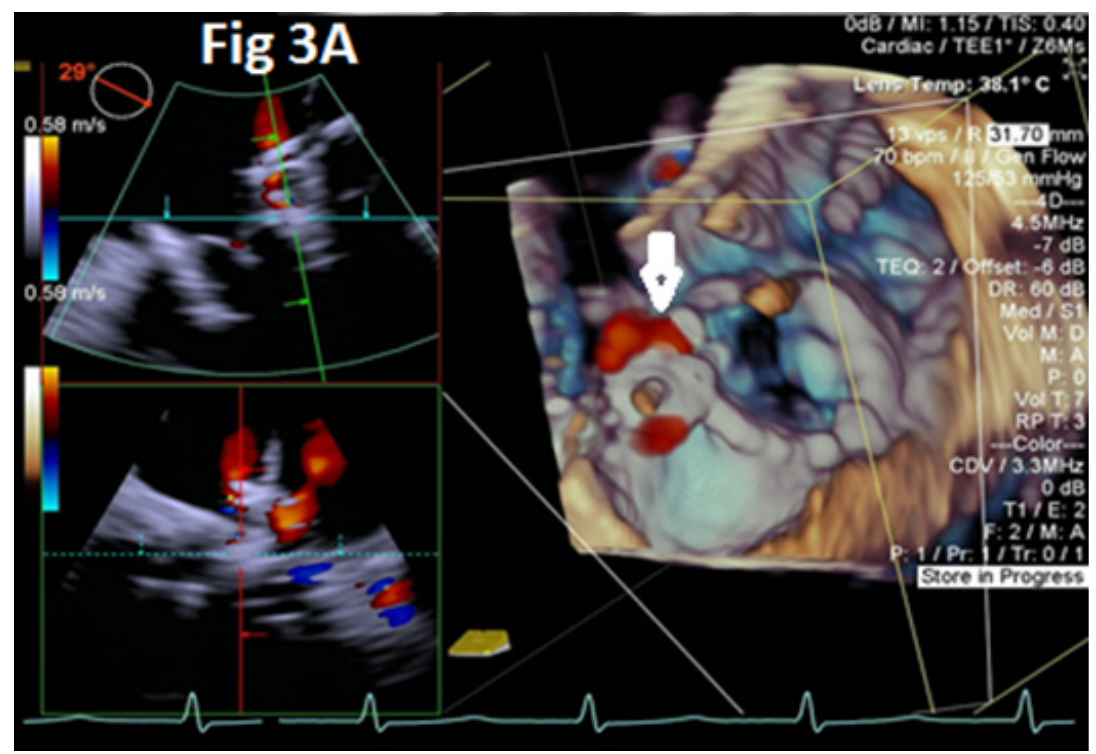

Citation: Abdulbasit M, Kozak M, Peterson B, et al. Tricuspid perivalvular leak closure through the open cells of Sapien 3 transcatheter heart valve. MOJ Clin Med Case Rep. 202I;I (3):79-83. DOI: 10.15406/mojcr.202I.II.00387 


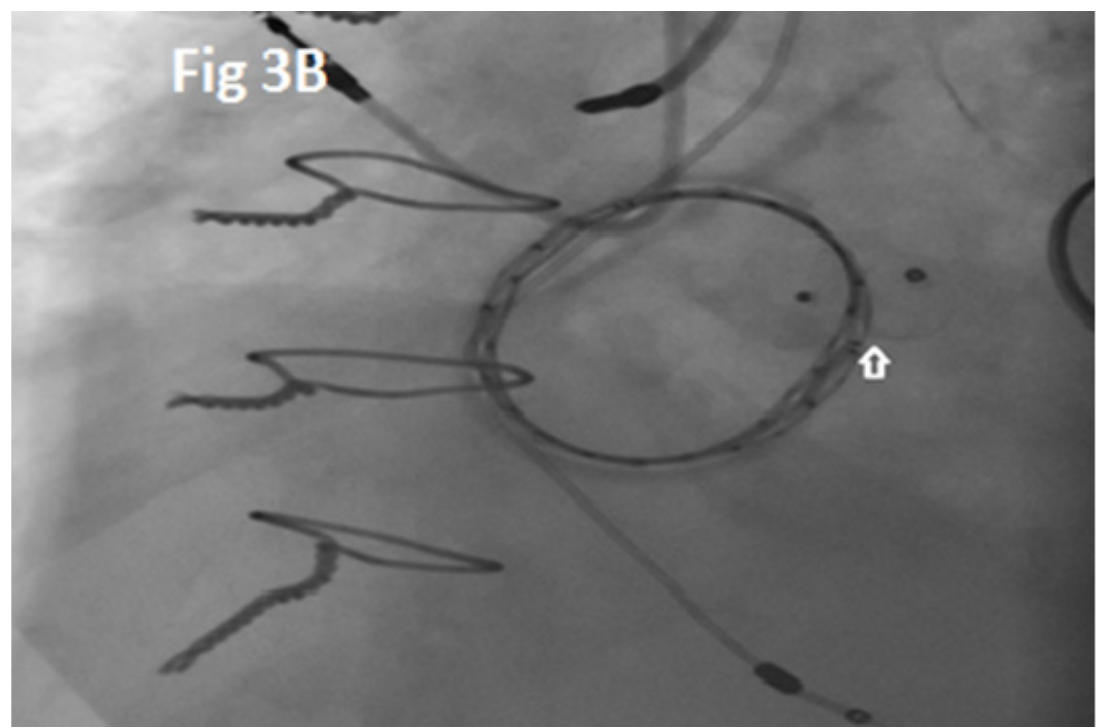

Figure 3 Successful implantation of Amplatzer ${ }^{\mathrm{TM}}$ Vascular plug II (white arrows).

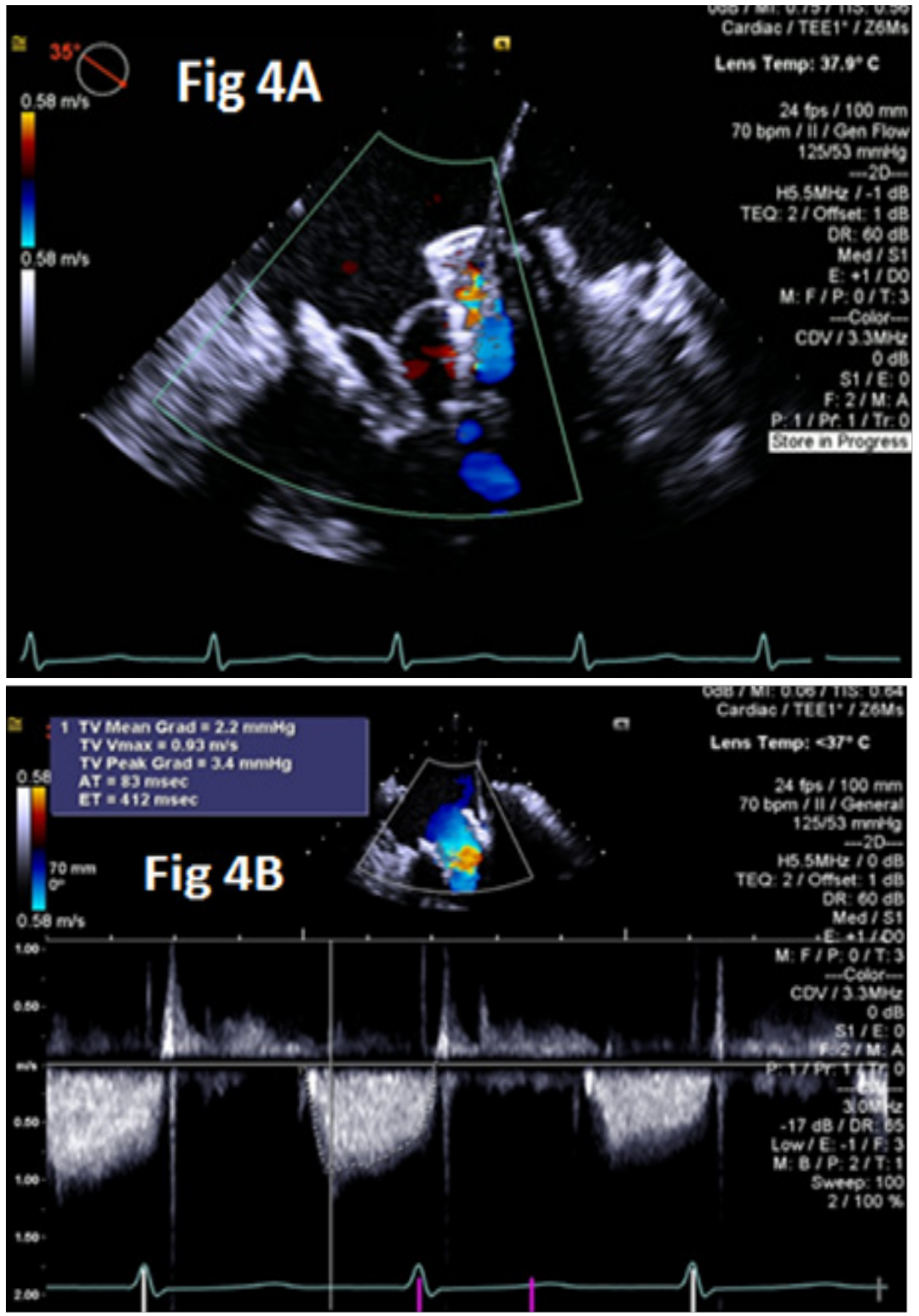

Citation: Abdulbasit M, Kozak M, Peterson B, et al. Tricuspid perivalvular leak closure through the open cells of Sapien 3 transcatheter heart valve. MOJ Clin Med Case Rep. 202I;I I(3):79-83. DOI: 10.15406/mojcr.202I.II.00387 


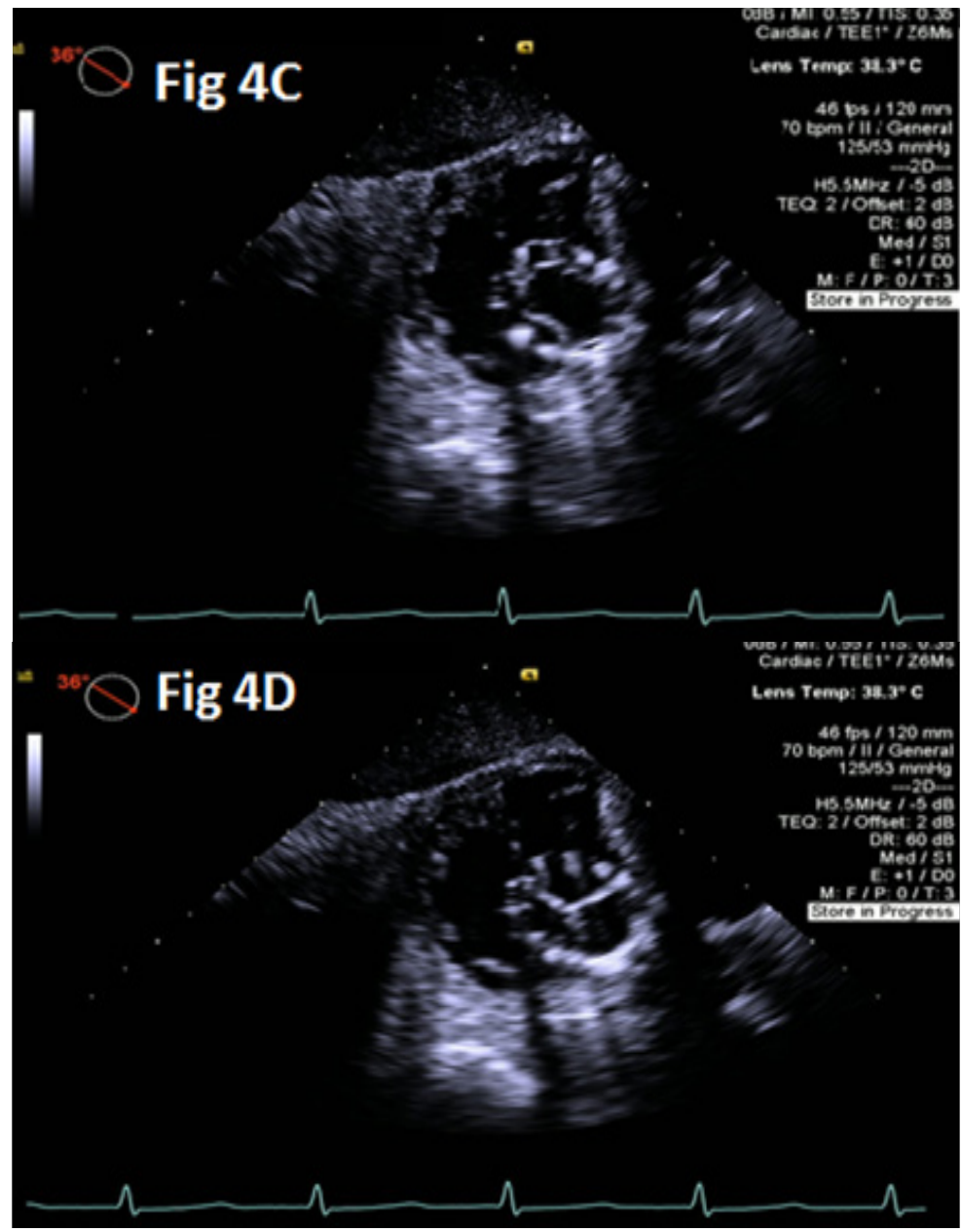

Figure 4 Post Amplatzer ${ }^{\mathrm{TM}}$ vascular plug II with mild residual PVL (4A), mildly elevated trans-S3 gradient (4B) and normal leaflet mobility of S3 (diastole 4C, Systole 4D).

Video 2 Mid esophageal view showing Amplatzer vascular plug II (AVP II) on septal side of the Sapien 3 valve.

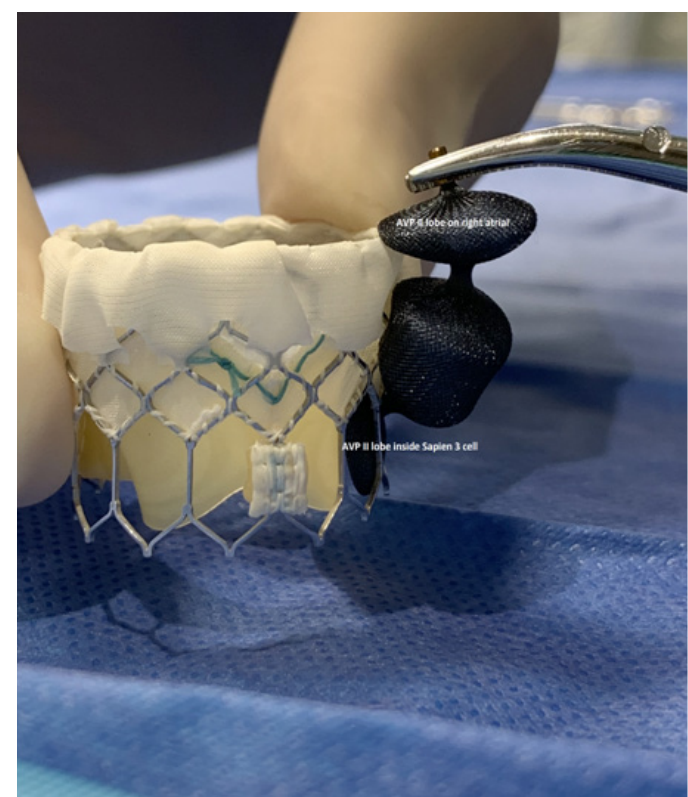

Figure 5 Amplatzer vascular plug II (AVP II) position in relation to Sapien 3 valve.

\section{Disclosure statement \& financial support}

Pradeep Yadav MD is a proctor and has accepted research grant from Edwards Life sciences. Pradeep Yadav MD is on the Speaker's Bureau of Abbott Vascular Inc. All other authors have no relationship relevant to the contents of this paper to disclose. No other authors have any financial conflict of interest to disclose.

\section{Acknowledgments}

None.

\section{References}

1. Peters, AC, Unger, E, Gong, FF, et al. Multimodality imaging to guide transcatheter treatment of severe degenerative tricuspid regurgitation with tricuspid valve-in-ring implantation and paravalvular leak closure. Echocardiography. 2020; 37(6):913-916.

2. Polonetsky O, Stelmashok V, Sevruk T, et al. Tricuspid paravalvular leak closure with a paravalvular leak device. Postepy Kardiol Interwencyjnej. 2017;13(3):273- 274 .

3. Mukherji A, Anantharaman R, Subramanyan R. Percutaneous closure of symptomatic large tricuspid paravalvular regurgitation using two muscular VSD occluders. Indian Heart J. 2017;69(3):334-337. 